\section{Evolução da notificação de violência sexual no Brasil de 2009 a 2013}

\author{
Trends in reporting of sexual violence in Brazil \\ from 2009 to 2013
}

\section{Evolución de notificaciones sobre violencia sexual en Brasil de 2009 a 2013}

\section{Resumo}

O objetivo foi analisar a evolução das notificações de violência sexual no Brasil entre 2009 e 2013, dando especial enfoque ao estupro. Realizou-se um estudo observacional de série temporal, analisando dados entre 2009 e 2013, provenientes do banco de dados secundários coletados do Sistema de Informação de Agravos de Notificação (SINAN). A variável independente referiu-se ao número de notificações de violência sexual entre 2009 e 2013, sendo esta comparada a diversas variáveis sociodemográficas, de violência e de saúde. Os dados foram analisados por modelo de regressão linear generalizada de Prais-Winsten, determinando as variações como estável, crescente ou decrescente pela análise do coeficiente de determinação ( $R^{2}$ de Pearson) e nível de significância $(p<0,05)$. As notificações de violência sexual tiveram maior variação positiva em: 10-19 anos (364\%), indígenas (520\%), da Região Sul (414\%), sexo feminino (331\%) e baixa escolaridade (343\%). Mais de 70\% das notificações foram estupros, enquanto um terço foi casos de repetição, com decréscimo de notificações acompanhadas de espancamento. As características do agressor mantiveram-se estáveis. O local de ocorrência modificou-se, com menos casos ocorridos em via pública. Por fim, a taxa de notificação estupro aumentou em 590\%, com tendência semelhante aos estupros dentro de casa e aumento da resolutividade dos atendimentos. O presente estudo evidencia o perfil sociodemográfico das vítimas de violência sexual notificadas no Brasil e o aumento da notificação de estupros atrelado aos casos em domicílio. Ainda, houve aumento da resolutividade das notificações.

Violência contra a Mulher; Delitos Sexuais; Estupro; Notificação Compulsória
Renato Simões Gaspar 1,2

Marina Uchoa Lopes Pereira 2

doi: 10.1590/0102-311X00172617

\author{
Correspondência \\ R. S. Gaspar \\ Institute of Cardiovascular and Metabolic Research, \\ University of Reading. \\ Reading / Berkshire - RG6 6AS, U.K. \\ renatosgaspar@gmail.com \\ 1 University of Reading, Reading, U.K. \\ 2 Universidade Federal do Maranhão, São Luís, Brasil.
}




\section{Introdução}

A violência é um fenômeno social que afeta a saúde coletiva, sendo considerada a quarta causa de morte na população em geral em 2015 1. Autores como Minayo \& Deslandes 2 historicamente apontam que a violência é produzida com base em problema social, advindo de fatos políticos, econômicos e culturais, devendo, portanto, ser encarada sob tal complexidade a fim de sustar seus efeitos deletérios.

Dentre os tipos de violência, destaca-se a violência sexual, sendo definida por qualquer ação em que uma pessoa em situação de poder e com uso de força física, coerção, intimidação ou influência psicológica, com ou sem uso de armas ou drogas, obriga outra pessoa a ter, presenciar ou participar de alguma interação sexual ou a utilizar, de qualquer modo, a sua sexualidade ${ }^{3}$. Atualmente, a violência sexual é reconhecida como um desafio no âmbito da saúde coletiva, sendo relatada em $63 \%$ das mulheres e $25 \%$ dos homens na literatura estadunidense 4. Já no Brasil, revisão de literatura identificou que até 40\% das mulheres e 35\% dos homens relataram alguma forma de agressão sexual no ano anterior ao estudo 5 .

A despeito da alta prevalência, a violência sexual deve ser encarada para além dos números, não apenas pela notória subnotificação deste tipo de violência 6,7 , mas também por causar consequências físicas e psicológicas nas vítimas 8 , como transtornos de ansiedade, depressão e abuso de substâncias, especialmente quando a violência ocorre na infância e adolescência ${ }^{9}$. Essas consequências têm continuação na vida adulta e causam impactos negativos na sociedade, tanto pela perspectiva individual 10 quanto comunitária e econômica 11 .

No Brasil, houve mudanças da legislação nas últimas décadas a fim de melhor tipificar a violência sexual como crime. Na década de 2000, foram realizadas modificações no Código Penal, por meio das Leis no 11.106/2005 12 e no 12.015/200913, pelas quais se alterou o conceito de estupro, que agora abrange também os casos de atentado violento ao pudor e foram incluídos ambos os sexos como possíveis vítimas de violência sexual.

Aliado às mudanças de conceitos e procurando entender melhor a complexidade do problema, adotou-se a estratégia de notificação universal e obrigatória dos casos de violência sexual. Em 2006, foi implantado o Sistema de Vigilância de Violências e Acidentes (VIVA), que teve especial atenção à violência doméstica e sexual, e contribuiu, por meio do Sistema de Informação de Agravos de Notificação (SINAN), com um importante banco de dados oficiais 14. Não obstante, estudos com dados provenientes do SINAN sobre violência sexual ainda são reduzidos.

Os profissionais de saúde são essenciais na identificação e intervenção de situações de violência sexual. São eles que fazem a notificação da violência, e para que haja um atendimento resolutivo, uma intervenção interdisciplinar se faz necessária. Dentre outras, a profilaxia de doenças sexualmente transmissíveis, a prevenção de uma gravidez indesejada e, sobretudo, o acolhimento, são medidas que contribuem para um bom atendimento à vítima. Por vezes, o encaminhamento para ambulatório específico ou para unidade básica de saúde também é necessário 15 .

Assim, tendo em vista a importância epidemiológica da violência sexual no mundo e no Brasil, é imperativo o estudo da evolução deste problema de saúde pública no Brasil que se valha de dados oficiais de notificação, ainda que eles não representem o número real de casos. Tais estudos buscam guiar a implementação de políticas públicas mais eficazes para o combate à violência. Sob nenhuma ótica este relato busca precisar taxas de violência sexual, mas dedica-se a esmiuçar a evolução das notificações e suas implicações à saúde coletiva. Portanto, o presente estudo objetivou analisar a evolução das notificações de violência sexual no Brasil entre 2009 e 2013, dando especial enfoque ao estupro.

\section{Materiais e métodos}

Realizou-se um estudo observacional de série temporal, no qual se coletaram todas as notificações de casos de violência sexual ocorridos entre janeiro de 2009 e dezembro de 2013, inseridas na base nacional do SINAN.

A variável independente, utilizada em todos os dados aqui apresentados, referiu-se ao número de casos positivos para violência sexual. As variáveis dependentes incluíram: região, idade, sexo, raça autorreferida, escolaridade, tipos de violência sexual (assédio sexual e estupro), meios de agressão utilizados (tortura, espancamento e com arma de fogo), violência de repetição, dados do agressor 
(suposto uso de álcool, amigo ou conhecido), local da ocorrência (via pública, residência) e setores de saúde (encaminhamento ambulatorial e internação hospitalar). A definição de cada uma das variáveis pode ser encontrada no instrutivo do SINAN 3.

Estupro foi definido como todos os casos positivos para estupro dentro dos casos de violência sexual, enquanto estupros dentro de casa referem-se aos casos de estupros ocorridos apenas em residência, conforme a subseção local dentre variáveis apresentadas pelo SINAN. Para o cálculo de variação dentro do período analisado, obteve-se o acréscimo em percentual do número de casos, tendo como base o primeiro ano analisado. O número de habitantes por região foi obtido em dados derivados dos censos de 2000 e 2010 e estimativas intercensitárias, disponibilizados pelo Instituto Brasileiro de Geografia e Estatística (IBGE).

As tabelas e figuras representam medidas de incidência, crescimento, tendência central e proporções. Todos os dados foram tabulados em Microsoft Excel (versão 2010 ou superior, https:// products.office.com/) e posteriormente analisados pelo Graphpad Prism 5.0 (https://www.graphpad. com/support/prism-5-updates/). Utilizou-se um modelo de regressão linear generalizada de PraisWinsten para analisar as tendências na série temporal. Isso permitiu determinar as variações como estável, crescente ou decrescente pela análise do coeficiente de determinação (R2 de Pearson) e nível de significância $(\mathrm{p}<0,05)$.

\section{Resultados}

$\mathrm{Na}$ Tabela 1, estão expostas as características sociodemográficas das vítimas e sua variação entre os anos de 2009 e 2013. Nota-se que houve aumento de notificação de violência sexual com relação a todas as variáveis. Destaca-se a Região Sul como a que demonstrou maior variabilidade, enquanto a Sudeste concentra o maior número de casos dentro do período analisado. Também foi observada maior variância com relação às vítimas com mais de 60 anos de idade, do sexo feminino, da raça indígena e com escolaridade menor de 8 anos.

A Tabela 2 mostra a frequência dos tipos de violência sexual, assédio sexual e estupro, com relação ao número total de casos. Os casos de estupro foram os mais frequentes dentro do período analisado, passando de 54,61\% em 2009 para 74,39\% em 2013, ainda que a análise de tendência não tenha corroborado o aumento.

Já a Tabela 3 traz a relação entre as características de violência sexual com relação ao número total de notificações. Dentre os meios de agressão empregados, houve diminuição da notificação relativa de espancamento, embora tenha estado presente em aproximadamente um terço dos casos. Além disso, foi notório o aumento da proporção de violência de repetição.

Ainda na Tabela 3, constata-se que, em todos os anos, mais da metade dos casos ocorreu em residência, conquanto observou-se diminuição significativa dos casos de violência sexual ocorridos em via pública. Cabe salientar que as demais circunstâncias de violência sexual analisadas mantiveram tendência estável dentro do período estudado.

Considerando tendência estável dos casos de estupro e violência sexual dentro de casa (Tabelas 1 e 3) e a relevância social e de saúde de tal tipo de violência sexual, buscou-se aprofundar a análise nos casos de estupro, expostos na Figura 1. Ocorreu significativa tendência de crescimento para a taxa de estupros e estupros dentro de casa/100 mil habitantes (Figura 1).

Essa mesma conexão entre tais variáveis se mostrou presente quando analisamos o total de casos de violência sexual (ver Material Suplementar: http://cadernos.ensp.fiocruz.br/csp/public_site/ arquivo/csp-1726-17-material-suplementar_4362.pdf). Portanto, o aumento das taxas de estupro se deu primariamente pelo acréscimo de 590\% nas notificações de casos ocorridos dentro de casa.

A Figura 2 mostra as frequências de encaminhamentos após o caso de violência sexual. Houve queda da proporção de notificações encaminhadas ao atendimento ambulatorial (Figura 2a), mas tendência estável para as notificações encaminhadas para internação hospitalar (Figura 2b), que manteve percentual de 5,64\%. Quando computados juntos, conclui-se que houve decréscimo significativo de 7,4\% da relação de notificações encaminhadas entre 2009 e 2013 (Figura 2c). Isso pode ter se dado por maior resolutividade do atendimento inicial (Figura $2 \mathrm{~d}$ ), que registrou aumento relativo de 4,88 para 9,69\% do total de notificações. 
Tabela 1

Evolução das notificações de violência sexual segundo características sociodemográficas. Brasil, 2009-2013.

\begin{tabular}{|c|c|c|c|c|}
\hline \multirow[t]{2}{*}{ Características sociodemográficas } & \multicolumn{2}{|c|}{ Notificações } & \multirow{2}{*}{$\begin{array}{c}\text { Variação 2009-2013 } \\
\text { (\%) }\end{array}$} & \multirow[t]{2}{*}{ Valor de $p$} \\
\hline & 2009 & 2013 & & \\
\hline \multicolumn{5}{|l|}{ Região } \\
\hline Norte & 1.112 & 4.371 & 393,07 & 0,0002 \\
\hline Nordeste & 1.084 & 4.261 & 393,08 & 0,0006 \\
\hline Sudeste & 3.321 & 8.376 & 252,21 & 0,0013 \\
\hline Sul & 1.165 & 4.829 & 414,50 & 0,0004 \\
\hline Centro-oeste & 812 & 2.592 & 319,21 & 0,0024 \\
\hline \multicolumn{5}{|l|}{ Idade (anos) } \\
\hline$<1-10$ & 2.541 & 7.651 & 301,10 & 0,0003 \\
\hline $10-19$ & 3.113 & 11.332 & 364,02 & 0,0001 \\
\hline $20-59$ & 1.782 & 5.238 & 293,93 & 0,0006 \\
\hline$>60$ & 58 & 203 & 350,00 & 0,0025 \\
\hline \multicolumn{5}{|l|}{ Sexo } \\
\hline Masculino & 1.053 & 3.074 & 291,92 & 0,0016 \\
\hline Feminino & 6.440 & 21.354 & 331,58 & 0,0002 \\
\hline \multicolumn{5}{|l|}{ Raça } \\
\hline Branca & 3.121 & 8.946 & 286,63 & 0,0010 \\
\hline Preta & 594 & 2.078 & 349,83 & 0,0010 \\
\hline Amarela & 73 & 166 & 227,39 & 0,0074 \\
\hline Parda & 2.713 & 9.918 & 365,57 & 0,0002 \\
\hline Indígena & 44 & 229 & 520,45 & 0,0090 \\
\hline \multicolumn{5}{|l|}{ Escolaridade (anos) } \\
\hline$<8$ & 2.888 & 9.924 & 343,62 & 0,0001 \\
\hline $8-12$ & 1.283 & 3.831 & 298,59 & 0,0006 \\
\hline$>12$ & 226 & 679 & 300,44 & 0,0068 \\
\hline Total & 7.494 & 24.429 & & \\
\hline
\end{tabular}

Tabela 2

Evolução dos tipos de violência sexual. Brasil, 2009-2013.

\begin{tabular}{lllllll}
\hline & \multicolumn{4}{c}{ \% de casos positivos/total de casos de violência sexual } & \multicolumn{2}{c}{ Valor de p } \\
& $\mathbf{2 0 0 9}$ & $\mathbf{2 0 1 0}$ & $\mathbf{2 0 1 1}$ & $\mathbf{2 0 1 2}$ & $\mathbf{2 0 1 3}$ & \\
\hline \multirow{2}{*}{ Assédio sexual } & 17,82 & 20,18 & 19,87 & 21,01 & 20,29 & 0,14 \\
Estupro & 54,61 & 68,79 & 72,30 & 74,24 & 74,39 & 0,0620 \\
\hline
\end{tabular}

\section{Discussão}

Os resultados evidenciaram aumento do número de notificações de violência sexual no Brasil. Aconteceu diminuição do número de casos associados a espancamento, porém foi notório o aumento do número de notificações de violência sexual de repetição. Também se encontrou aumento das notificações de violência sexual ocorrida em residência. Destaca-se que a curva de número de casos de estupro foi crescente no decorrer dos anos e seguiu padrão semelhante à curva de número de estupros que ocorreram dentro de casa. Apesar da elevação dos números de notificações, constatou-se diminuição dos encaminhamentos de saúde, possivelmente devido à maior resolução no serviço que prestou o atendimento inicial, tornando desnecessário o encaminhamento. 
Tabela 3

Características das notificações de violência sexual. Brasil, 2009-2013.

\begin{tabular}{|c|c|c|c|c|c|c|}
\hline & \multicolumn{5}{|c|}{ \% de casos positivos/total de casos de violência sexual } & \multirow[t]{2}{*}{ Valor de $p$} \\
\hline & 2009 & 2010 & 2011 & 2012 & 2013 & \\
\hline \multicolumn{7}{|c|}{ Meios de agressão utilizados } \\
\hline Tortura & 6,17 & 6,58 & 5,99 & 5,86 & 5,30 & 0,0795 \\
\hline Espancamento & 41,25 & 39,22 & 38,87 & 38,65 & 37,57 & 0,0210 \\
\hline Com arma de fogo & 7,79 & 5,76 & 5,41 & 5,26 & 4,95 & 0,0587 \\
\hline \multicolumn{7}{|c|}{ Característica da violência sexual } \\
\hline De repetição & 33,73 & 34,77 & 35,09 & 35,40 & 35,97 & 0,0060 \\
\hline \multicolumn{7}{|l|}{ Dados do agressor } \\
\hline Suposto uso de álcool & 21,96 & 21,73 & 22,96 & 21,16 & 20,74 & 0,3222 \\
\hline Amigo ou conhecido & 23,97 & 25,50 & 24,84 & 26,01 & 25,52 & 0,1667 \\
\hline \multicolumn{7}{|l|}{ Local da ocorrência } \\
\hline Via pública & 16,54 & 16,19 & 14,93 & 14,28 & 14,67 & 0,0351 \\
\hline Residência & 53,68 & 56,18 & 58,26 & 58,99 & 57,95 & 0,0730 \\
\hline
\end{tabular}

\section{Figura 1}

Notificações de estupro e estupro dentro de casa por 100 mil habitantes. Brasil, 2009-2013.
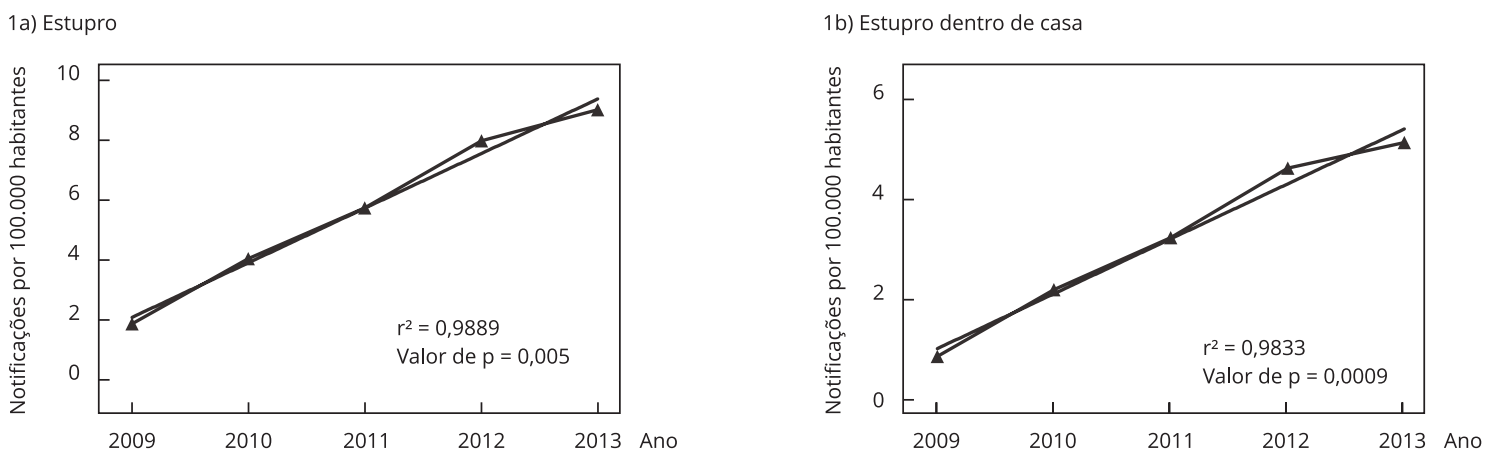

Fonte: dados coletados do Sistema de Informação de Agravos de Notificação (SINAN), correspondentes às notificações de estupro e estupros ocorridos em residência e do Instituto Brasileiro de Geografia e Estatística (IBGE) correspondentes a censos populacionais no período estudado. Nota: (1a) Proporção das notificações de estupro em relação ao total de notificações de violência sexual por 100 mil habitantes e correspondente linha de regressão linear. (1b) Proporção das notificações de estupros dentro de casa em relação ao total de estupros por 100 mil habitantes e sua correspondente linha de regressão linear.

O objetivo deste trabalho não foi definir taxas precisas dos casos de violência sexual, mas sim estudar o comportamento das notificações no período de 2009-2013. Com isso, apesar de as notificações de violência sexual terem triplicado no período do presente estudo, o fato pode ter se dado pelo aumento real do número de casos, por maior conscientização das vítimas e/ou profissionais de saúde que fazem o acolhimento ou ainda por melhor estruturação e quantidade de unidades notificantes.

Quanto às regiões do país, o Sudeste teve mais casos notificados em todos os anos. Isso pode ter se dado não só por esta região concentrar maior número de pessoas, mas por possuir maior número de unidades notificantes. Estudo anterior corrobora tais dados ao demonstrar que, no ano de 2010, São Paulo foi o estado com maior número total de notificações de casos de violência sexual em crianças, 
Figura 1

Encaminhamentos para setores de saúde em notificações de violência sexual. Brasil, 2009-2013.

2a) Encaminhamento ambulatorial

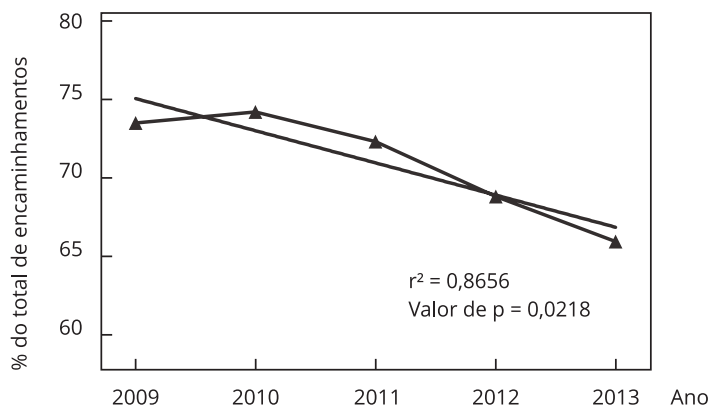

2c) Total do encaminhamento

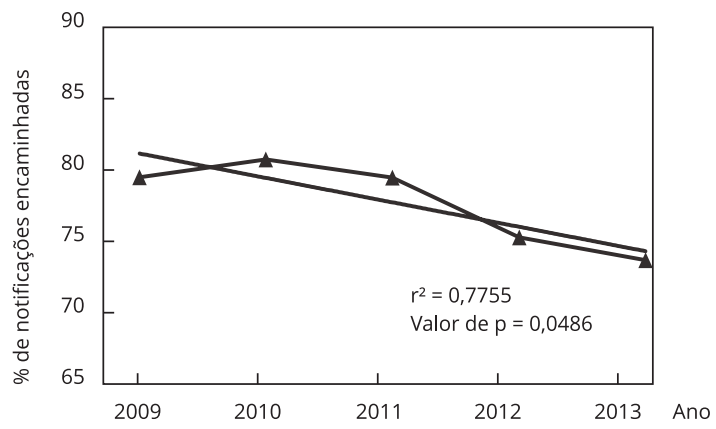

2b) Internação hospitalar

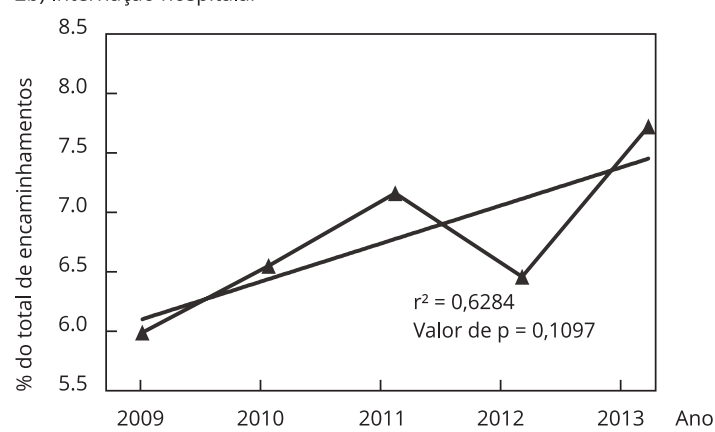

2d) Notificações resolvidas

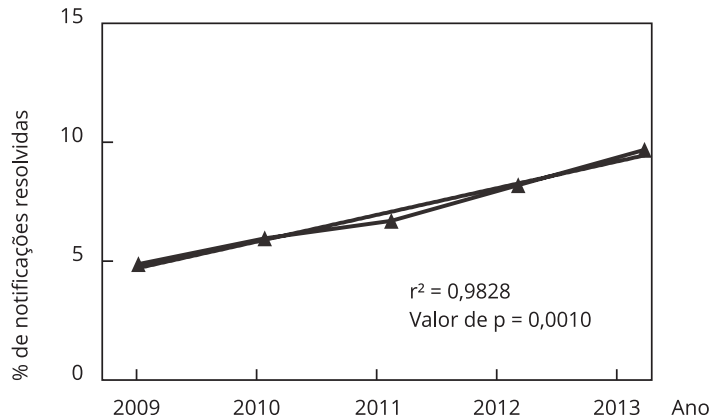

SINAN: Sistema de Informação de Agravos de Notificação.

Fonte: dados coletados do SINAN correspondentes às notificações encaminhadas a setores de saúde em relação ao total de encaminhamentos. Notificações resolvidas consistiram de casos preenchidos como "não se aplica" conforme instrutivo do SINAN.

Nota: (2a) Proporção de notificações encaminhadas a atendimento ambulatorial em relação ao total de notificações de violência sexual encaminhada e linha de regressão linear. (2b) Proporção de notificações de internação hospitalar em relação ao total de notificações encaminhadas e linha de regressão linear. (2c) Proporção de notificações encaminhadas a ambulatórios e internação hospitalar em relação ao total de notificações e linha de regressão linear. (2d) Proporção de notificações preenchidas como "não se aplica" e linha de regressão linear.

sendo também o estado com maior número de unidades de saúde notificantes 16. Além disso, é possível que nesta região haja maior busca ativa e mais conscientização dos profissionais e trabalhadores com relação à importância da notificação, como demonstrado por de Sousa 14.

Já com relação à escolaridade da vítima, a maioria dos casos ocorreu em pessoas com menos de 8 anos de estudo, o que era esperado porque a maioria das vítimas tinha menos de 14 anos e o instrutivo do SINAN orienta o profissional de saúde a preencher "não se aplica" na categoria escolaridade a vítimas menores de 6 anos de idade e pessoas com comprometimento mental severo ${ }^{3}$. Assim, é provável que, dentre as vítimas adultas, haja maior número de casos de violência sexual contra pessoas com menor escolaridade. Tal ideia é corroborada por estudo recente que analisou as notificações de violência sexual em Santa Catarina, Brasil 17. Por conseguinte, o presente estudo reforça que o número de notificações de violência sexual vem aumentando mais entre pessoas de baixa escolaridade, possivelmente pelos avanços na conscientização dessa população.

Das notificações, $86 \%$ dos casos de violência sexual foram contra a mulher, revelando uma marcante violência de gênero. A pouca notificação de violência sexual contra homens se dá em parte pela masculinidade tóxica que, por receio de ter sua virilidade questionada, não buscam os serviços 
de saúde após terem sofrido violência sexual. Ainda que homens sofram este tipo de violência, as mulheres são as principais vítimas em todas as faixas etárias. Esse é um reflexo das origens históricas da violência contra a mulher, relacionadas à posição em que homens e mulheres supostamente devem assumir na sociedade. O patriarcado reforça a ideia de que o homem possui a autoridade máxima, seja como pai ou como parceiro, e é ainda muito presente nas sociedades ocidentais 8 . Tais noções de sociedade contribuem para que haja uma repetição desses atos de agressão, particularmente quando o agressor é alguém próximo ou conhecido da vítima, já que a existência de vínculo leva a vítima, no geral, a adiar mais a procura de ajuda 18.

Ressalta-se que o agressor geralmente possui um relacionamento de confiança e poder com a vítima de violência sexual, sendo pai, padrasto ou amigo da família 19,20,21. A proximidade que o agressor tem facilita sua abordagem, propiciando a recorrência da violência, o que se correlaciona com os achados desta pesquisa, em que um terço dos casos foi de violência de repetição. Outro achado que ratifica esta ideia é o fato de que $63 \%$ dos casos ocorreram em crianças de até 14 anos. Nessa idade, não há ainda desenvolvimento completo e percepção por parte da criança ou do adolescente de que o ocorrido se trata de violência 22 .

Apesar desse padrão crescente de número de notificações, não foi verificado aumento do número de casos de violência sexual por amigos/conhecidos, ainda que outras análises tenham relatado altos índices 23,24. É importante frisar que dentre eles não estão inclusos parceiro ou pai/mãe, mas sim outros conhecidos, como um vizinho ou professor. É possível que esse padrão estático tenha ocorrido porque, dentre o total das vítimas de violência sexual, a maioria é de crianças e adolescentes de até 14 anos. Reforça-se que tais vítimas, com frequência, não buscam ajuda no período em que sofrem a violência, mas sim anos depois, por culpa ou medo, ou por não terem consciência do que exatamente é um ato de violência sexual 25,26,27.

Apesar do aumento de notificações de violência sexual, verificou-se diminuição do número de casos em que foi utilizado espancamento ou tortura, indicando que atualmente é menos provável que o agressor se utilize da força física durante a agressão. Como dito anteriormente, o agressor se utiliza da confiança e autoridade sobre a vítima, muitas vezes não necessitando de meios de violência física para perpetrar a violência sexual. Tendo em vista que a maioria dos casos ocorreu na residência, em menores de 14 anos e foi uma violência de repetição, é notório que os agressores tendem a utilizar mais violência psicológica como meio de coerção 28 e, portanto, servem como justificativa para a diminuição desses meios de agressão.

Com relação ao tipo de violência sexual, observou-se aumento da proporção de estupros. Com a mudança legislativa ocorrida em 2009, em que os casos de atentado violento ao pudor passaram a ser considerados como estupro 13 , a elevação já era esperada.

Mesmo que não tenha havido tendência crescente na proporção de estupros dentro dos casos de violência sexual, constatou-se aumento significativo da taxa de estupro (Figura 1), taxa essa correlacionada aos casos de estupro dentro de casa. Pesquisas anteriores utilizando dados do SINAN já relataram alta prevalência de estupros em residência $21,29,30$. No entanto, não foram encontrados estudos anteriores abordando a relação entre estupro e estupro dentro de casa que usem dados do SINAN. Sendo assim, o presente trabalho aprofunda a análise da evolução dos casos de estupro no Brasil pela utilização dessa base de dados, demonstrando que o aumento dos casos se dá primariamente por aumento das notificações ocorridas dentro da residência (Figura 1 e Material Suplementar: http:// cadernos.ensp.fiocruz.br/csp/public_site/arquivo/csp-1726-17-material-suplementar_4362.pdf). Tal correlação enseja o debate quanto à implementação de políticas públicas que visem prevenir ou conter os casos de estupro ocorridos dentro de residência e estimular ainda mais a notificação deles.

Com o aumento do número de notificações, esperava-se acréscimo concomitante dos encaminhamentos a serviços de saúde. Paradoxalmente, dados expostos na Figura 2 evidenciam o contrário, com diminuição significativa desses encaminhamentos e aumento da resolutividade das notificações. Tendo em vista a diminuição dos casos de violência sexual concomitantes à tortura e ao espancamento, parece cabível supor que tal efeito poderia diminuir o número de internações hospitalares, mas ainda não explicaria o decréscimo de seguimento dessas vítimas em serviços de saúde.

A fim de elucidar os motivos da diminuição de encaminhamentos, destaca-se a incapacidade de o sistema de saúde atender a demanda, o que é reforçado pelo fato de que em 2010 o número de hospitais em que se realizavam abortos legais estava em declínio, sugerindo retrocesso ao cuidado dessas 
vítimas 31 . Além disso, é possível ter ocorrido diminuição de casos de violência sexual que requeiram atendimentos mais complexos. Porém, tendo em vista a melhora socioeconômica e de estruturação de serviços, é possível que a diminuição de encaminhamentos tenha ocorrido por uma melhor resolutividade no atendimento dos casos, conforme exposto na Figura $2 \mathrm{~d}$.

Como todo trabalho científico, é preciso ter clareza das limitações do presente estudo. Primeiramente, é provável haver subnotificação, já que se estima que apenas cerca de $10 \%$ do total de casos de violência sexual sejam notificados 20 , além de erros presentes nas fichas de notificação, causados pela dificuldade de preenchimento aliada à falta de sensibilização dos profissionais que as preenchem 14 . Apesar disso, reforça-se a importância de realizar estudos mediante dados oficiais de notificação. Dessa forma, os dados aqui apresentados ressaltam, sobretudo, a necessidade de desenvolvimento de processos de educação continuada para que os profissionais estejam mais preparados para identificar um caso e notificá-lo da maneira correta. Logo, as conclusões científicas poderão ser concretizadas a ponto de contribuírem com a construção de políticas públicas mais eficazes.

O presente trabalho buscou aprofundar os conhecimentos acerca das notificações de violência sexual no Brasil. Com maior variação na Região Sul, em mulheres, em indígenas, maiores de 60 anos e com baixa escolaridade, é possível traçar o perfil sociodemográfico das vítimas. Mais de um terço foi de casos de repetição, com redução nos casos associados a espancamento, aumento das taxas de estupro e evidente correlação aos casos dentro de casa. Já a diminuição dos encaminhamentos de saúde aponta para maior resolutividade no atendimento. Por fim, tais achados permitem identificar dificuldades no enfrentamento do problema e propor soluções para a paulatina contenção dos casos de violência sexual no Brasil.

\section{Colaboradores}

R. S. Gaspar e M. U. L. Pereira contribuíram com o desenho, coleta de dados, análise e escrita do artigo.

\section{Agradecimentos}

Os autores agradecem ao Laboratório de Ensino e Pesquisa Experimental em Fisiologia da Universidade Federal do Maranhão por ceder o espaço necessário para a realização da pesquisa.

\section{Referências}

1. Malta DC, França E, Abreu DMX, Perillo RD, Salmen MC, Teixeira RA, et al. Mortality due to noncommunicable diseases in Brazil, 1990 to 2015, according to estimates from the Global Burden of Disease study. São Paulo Med J 2017; 135:213-21.

2. Minayo MCS, Deslandes SF. A complexidade das relações entre drogas, álcool e violência. Cad Saúde Pública 1998; 14:35-42.

3. Ministério da Saúde. Viva: instrutivo de notificação de violência doméstica, sexual e outras violências. Brasília: Ministério da Saúde; 2011.

4. Breiding MJ, Smith SG, Basile KC, Walters ML, Chen J, Merrick MT. Prevalence and characteristics of sexual violence, stalking, and intimate partner violence victimization: national intimate partner and sexual violence survey, United States, 2011. MMWR Surveill Summ 2014; 63:1-18.

5. Winzer L. Frequency of self-reported sexual aggression and victimization in Brazil: a literature review. Cad Saúde Pública 2016; 32:e00126315. 
6. Moraes CL, Cabral CS, Heilborn ML. Magnitude e caracterização de situações de coerção sexual vivenciadas por jovens de três grandes capitais brasileiras: Porto Alegre, Rio de Janeiro e Salvador. Cad Saúde Pública 2006; 22:1493-504.

7. Schraiber LB, D'Oliveira AFPL, Couto MT, Hanada H, Kiss LB, Durand JG, et al. Violência contra mulheres entre usuárias de serviços públicos de saúde da Grande São Paulo. Rev Saúde Pública 2007; 41:359-67.

8. Duarte MC, Fonseca RM, Souza V, Pena ED. Gender and violence against women in nursing literature: a review. Rev Bras Enferm 2015; 68:297-303.

9. Hohendorff JV, Habigzang LF, Koller SH. Psicoterapia para crianças e adolescentes vítimas de violência sexual no sistema público: panorama e alternativas de atendimento. Psicol Ciênc Prof 2015; 35:182-98.

10. Duarte MC, Fonseca RMGS, Souza V, Pena ED. Gênero e violência contra a mulher na literatura de enfermagem: uma revisão. Rev Bras Enferm 2015; 68:325-32.

11. Post LA, Mezey NJ, Maxwell C, Wibert WN. The rape tax:tangible and intangible costs of sexual violence. J Interpers Violence 2002; 17:773-82.

12. Brasil. Lei no 11.106 , de 28 de março de 2005 . Altera os arts. 148, 215, 216, 226, 227, $231 \mathrm{e}$ acrescenta o art. 231-A ao Decreto-Lei no 2.848, de 7 de dezembro de 1940, (2005). Diário Oficial da União 2005; 29 mar.

13. Brasil. Lei no 12.015, de 7 de agosto de 2009. Altera o Título VI da Parte Especial do Decreto-Lei no 2.848, de 7 de dezembro de 1940 Código Penal, e o art. 1o da Lei no 8.072, de 25 de julho de 1990, Altera o Título VI da Parte Especial do Decreto-Lei no 2.848, de 7 de dezembro de 1940 - Código Penal, e o art. 1o da Lei no 8.072, de 25 de julho de 1990, que dispõe sobre os crimes hediondos, nos termos do inciso XLIII do art. 5o da Constituição Federal e revoga a Lei no 2.252, de 1o de julho de 1954, que trata de corrupção de menores. Diário Oficial da União 2009; 10 ago.

14. Sousa MH, Bento SF, Osis MJ, Ribeiro MP, Faundes A. Filling out the compulsory notification in health services that care for women who suffer from sexual violence. Rev Bras Epidemiol 2015; 18:94-107.

15. Vilela LF. Manual para atendimento às vítimas de violência na rede de saúde pública do Distrito Federal. Brasília: Secretaria de Estado de Saúde do Distrito Federal; 2009.

16. Assis SG, Avanci JQ, Pesce RP, Pires TO, Gomes DL. Notificações de violência doméstica, sexual e outras violências contra crianças no Brasil. Ciênc Saúde Coletiva 2012; 17:2305-17.

17. Delziovo CR, Bolsoni CC, Nazario NO, Coelho EBS. Características dos casos de violência sexual contra mulheres adolescentes e adultas notificados pelos serviços públicos de saúde em Santa Catarina, Brasil. Cad Saúde Pública 2017; 33:e00002716.
18. Acosta DF, Amarijo CL, Silva CD, de Oliveira Gomes VL, Cazeiro CC. Violência sexual: da denúncia à criminalização do agressor. Rev Enferm UERJ 2016; 24:e11518.

19. Rates SM, Melo EM, Mascarenhas MD, Malta DC. Violence against children: an analysis of mandatory reporting of violence, Brazil 2011. Ciênc Saúde Coletiva 2015; 20:655-65.

20. Cerqueira D, Coelho DSC. Estupro no Brasil: uma radiografia segundo os dados da saúde (versão preliminar). Brasília: Instituto de Pesquisa Econômica Aplicada; 2014. (Nota Técnica, 11).

21. Mascarenhas MDM, Sinimbu RB, Silva MMA, Carvalho MGO, Santos MR, Freitas MG. Caracterização das vítimas de violência doméstica, sexual e/ou outras violências no Brasil 2014. Saúde Foco (Impr, Rio J.) 2016; 1(1). https://smsrio.org/revista/index.php/revsf/ article/view/199.

22. Martins CBG, Jorge MHPM. Abuso sexual na infância e adolescência: perfil das vítimas e agressores em município do sul do Brasil. Texto \& Contexto Enferm 2010; 19:246-55.

23. Moreira GAR, Soares PS, Farias FNR, de Souza Vieira LJE. Notificações de violência sexual contra a mulher no Brasil. Rev Bras Promoç Saúde (Impr.) 2015; 28:327-36.

24. Silva MCM, Brito AM, Araújo AL, Abath MB. Caracterização dos casos de violência física, psicológica, sexual e negligências notificados em Recife, Pernambuco, 2012. Epidemiol Serv Saúde 2013; 22:403-12.

25. Paine ML, Hansen DJ. Factors influencing children to self-disclose sexual abuse. Clin Psychol Rev 2002; 22:271-95.

26. O'Leary P, Easton SD, Gould N. The effect of child sexual abuse on men: toward a male sensitive measure. J Interpers Violence 2017; 32:423-45.

27. Mathews S, Hendricks N, Abrahams N. A psychosocial understanding of child sexual abuse disclosure among female children in South Africa. J Child Sex Abus 2016; 25:636-54.

28. Leite FMC, Amorim MHC, Wehrmeister FC, Gigante DP. Violence against women, Espirito Santo, Brazil. Rev Saúde Pública 2017; 51:33.

29. Silva MCM, Brito AM, Araújo AL, Abath MB. Caracterização dos casos de violência física, psicológica, sexual e negligências notificados em Recife, Pernambuco, 2012. Epidemiol Serv Saúde 2013; 22:403-12.

30. Cerqueira D, Coelho DSC, Ferreira H. Estupro no Brasil: vítimas, autores, fatores situacionais e evolução das notificações no sistema de saúde entre 2011 e 2014. Rio de Janeiro: Instituto de Pesquisa Econômica Aplicada; 2017. (Texto para Discussão, 2313)

31. Lima CA, Deslandes SF. Violência sexual contra mulheres no Brasil: conquistas e desafios do setor saúde na década de 2000. Saúde Soc 2014; 23:787-800. 


\section{Abstract}

The objective was to analyze the trends in reporting of sexual violence in Brazil from 2009 to 2013, with special emphasis on rape. An observational times series study was conducted, analyzing data from 2009 to 2013 coming from a secondary database of the Brazilian National Information System for Notificable Diseases (SINAN). The independent variable was the number of reports of sexual violence from 2009 to 2013, which was compared to several sociodemographic, violence-related, and health-related variables. The data were analyzed with a Prais-Winsten generalized linear regression model, determining the variations as stable, upward, or downward by analysis of the coefficient of determination (Pearson's $R 2$ ) and level of significance set at $p<0.05$. The reports of sexual violence showed greater positive variation in the 10-19-year age bracket (364\%), indigenous individuals (520\%), in the South of Brazil (414\%), females (331\%), and individuals with low schooling (343\%). More than $70 \%$ of the reports were rapes, while one-third were repeat offenses, with a downward trend in reports of sexual violence accompanied by beating. The aggressors' characteristics remained stable. The site of violence changed, with fewer cases occurring on public byways. Finally, the reporting rate for rape increased by 590\%, with a similar trend in rapes in the household and an increase in case resolutions. The study reveals the sociodemographic profile of cases of sexual violence reported in Brazil and an increase in reported rapes, especially inside the household. There was also an increase in case resolution.

Violence Against Women; Sex Offenses; Rape; Mandatory Reporting

\section{Resumen}

El objetivo de este trabajo fue analizar la evolución de las notificaciones sobre violencia sexual en Brasil entre 2009 y 2013, otorgando especial relevancia a la violación. Se realizó un estudio observacional de carácter temporal, analizando datos entre 2009 y 2013, provenientes de un banco de datos secundarios, recabados por el Sistema de Información Nacional sobre Enfermedades de Notificación Obligatoria (SINAN por sus siglas en portugués). La variable independiente se refirió al número de notificaciones sobre violencia sexual entre 2009 y 2013, siendo esta última comparada con diversas variables sociodemográficas, de violencia y de salud. Los datos se analizaron mediante un modelo de regresión lineal generalizado de Prais-Winsten, determinando las variaciones como estables, crecientes o decrecientes por el análisis del coeficiente de determinación ( $R 2$ de Pearson) y el nivel de significancia $(p<0,05)$. Las notificaciones de violencia sexual contaron con una mayor variación positiva en: 10-19 años (364\%), indígenas (520\%), de la región Sur (414\%), sexo femenino (331\%) y baja escolaridad (343\%). Más del $70 \%$ de las notificaciones fueron violaciones, mientras que un tercio fueron casos de repetición, con un descenso de las notificaciones acompañadas de palizas. Las características del agresor se mantuvieron estables. El lugar de ocurrencia fue modificado, hubo menos casos que se produjeron en vía pública. Finalmente, la tasa de notificación de violación aumentó un 590\%, con una tendencia semejante a las violaciones dentro de casa y al aumento de la resolutividad de las atenciones. El presente estudio evidencia el perfil sociodemográfico de las víctimas de violencia sexual notificadas en Brasil y el aumento de la notificación de violaciones, relacionado con casos en domicilios. Asimismo, hubo un aumento de la resolutividad de las notificaciones.

Violencia Contra la Mujer; Delitos Sexuales; Violación; Notificación Obligatoria

Recebido em 04/Out/2017

Versão final reapresentada em 23/Jun/2018 Aprovado em 12/Jul/2018 